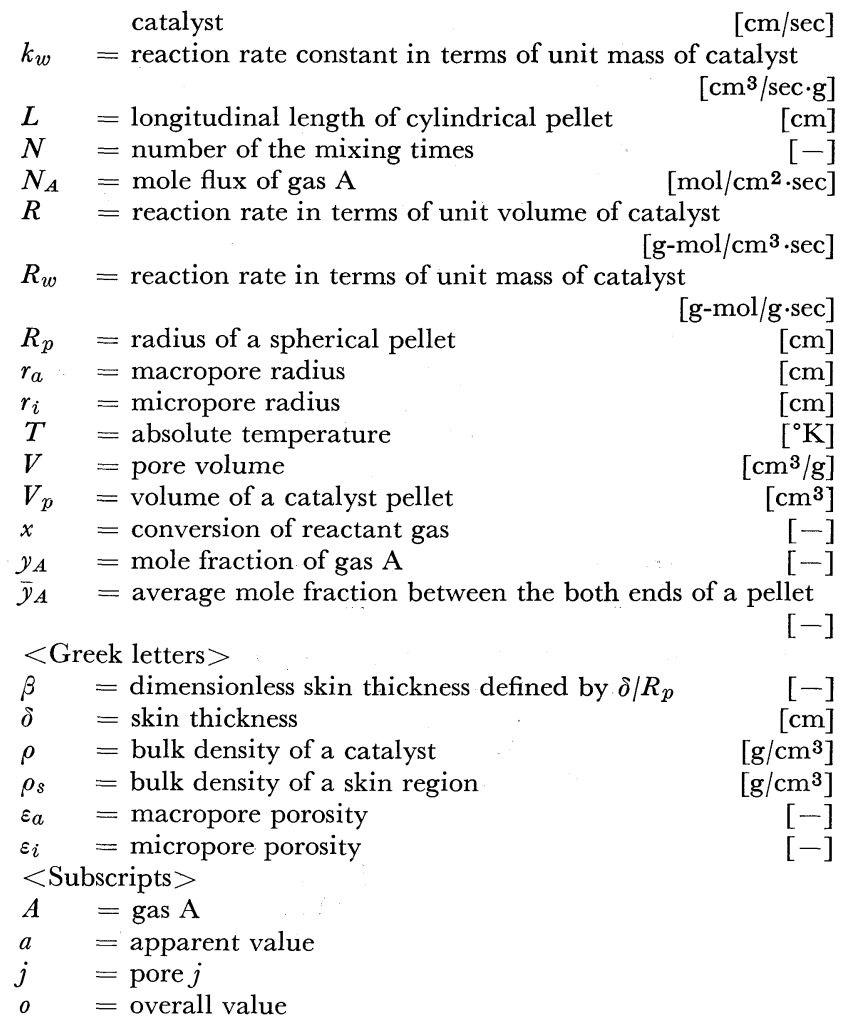

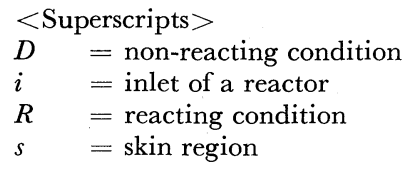

\section{Literature Cited}

1) Aris, R.: Chem. Eng. Sci., 6, 262 (1957)

2) Echigoya, E. and M. Iwasaki: Preprint of the 5th Symposium on Reaction Engineering, the Soc. Chem. Engrs., Japan, p. 31 (1965)

3) Foster, R. N. and J. B. Butt: A. I. Ch. E. J., 12, 180 (1968)

4) Kasaoka, S. and U. Sakata: J. Chem. Eng. Japan, 2, 138 (1968)

5) Korneichuk, G. P., Ya. V. Zhilailo, V. A. Roiter and I. P. Garkavenko: Zhur. Fiz. Khim., 29, 1073 (1955)

6) Otani, S. and J. M. Smith: J. Catalysis, 5, 332 (1966)

7) Petersen, E. E. and J. R. Balder: J. Catalysis, 11, 195, 202 (1968)

8) Raja Rao, M. and J. M. Smith: A. I. Ch. E. J., 10, 293 (1964)

9) Satterfield, C. N. and P. J. Cadle: Ind. Eng. Chem., Process Design \& Devel., 7, 256 (1968)

10) Thiele, E. W.: Ind. Eng. Chem., 31, 916 (1939)

11) Wakao, N. and J. M. Smith: Chem. Eng. Sci., 17, 825 (1962)

12) Wakao, N. and T. Funaki: Kagaku Kōgaku, 31, 485 (1967)

13) Wakao, N., H. Kimura, and M. Shibata: J. Chem. Eng. Japan, 2, 51 (1969)

14) Wakao, N. and J. M. Smith: Ind. Eng. Chem., Fundamentals, 3, $123(1964)$

15) Weisz, P. B. and A. B. Schwartz: J. Catalysis, 1, 399 (1962)

16) Wicke, E. and R. Kallenbach: Kolloid Z., 97, 135 (1941)

\title{
OPERATING RANGE OF FALLING FILM COLUMN IN VACUUM DISTILLATION*
}

\author{
Tsuyoshi MUNAKATA and Akira MATSUDA \\ Department of Chemical Engineering, Kyushu University, \\ Fukuoka, Japan
}

\begin{abstract}
It is pointed out that both the sonic velocity limit at the top(SVL) and the top pressure limit (TPL) should be considered as upper limits of vapor flow rate in a falling film column under vacuum distillation. These limits are represented in dimensionless forms and graphically, based on pressure drop equations involving a compressibility effect. Discussions are made also on the vapor Reynolds numbers corresponding to flooding and to the minimum wetting rate of reflux, and the effective operating range is illustrated.
\end{abstract}

\section{Introduction}

The falling film column is said to be applicable to vacuum distillation because of its low pressure drop and therefore its high allowable vapor load, but the operating limits are left uncertain due to insufficient

* Received on April 3, 1972.

Presented at the 37th Annual Meeting of the Soc. of Chem. Engrs., Japan at Nagoya (Apr., 1972). experimental and theoretical investigation.

The purpose of this paper is to predict how these limits vary with the operating pressure throughout a range from atmospheric to vacuum, possibly in a generalized manner.

\section{Sonic Velocity Limit at the Golumn Top (SVL)}

The upper limit of vapor load in a falling film column should be controlled by flooding if the oper- 
ating pressure is atmospheric, but under vacuum a different phenomenon arises. Under a constant bottom pressure $p_{1}$ to increase the vapor throughput, the top pressure $p_{2}$ (see Fig. 1) must be lowered, but after $p_{2}$ reaches a certain level it is impossible to lower $p_{2}$ further and a maximum vapor throughput is obtained depending on this value of $p_{2}$, even if the pressure at the overhead condenser or the vacuum generator is brought to a still lower value. At this time the vapor velocity: at the column top becomes comparable to the sonic velocity. Hence this limit may be called the sonic velocity limit at the column top (SVL).

\section{Column Top Pressure Limit (TPL)}

When the ratio of the column height to diameter is large, $p_{2}$ corresponding to SVL becomes very small compared with $p_{1}$. In such circumstances, a minimum $p_{2} / p_{1}$ value will be proposed as another limit of $p_{2}$, because extra conditions of higher vacuum are required for the equipment and the vacuum generating system if $p_{2}$ is too small compared with $p_{1}$. This limit may be called the column top pressure limit (TPL).

\section{Pressure Drop Equations}

It is evident from the foregoing discussion that the pressure drop equation must be one applicable to a high velocity compressible flow.

In a continuum flow region, including both turbulent and laminar flows, a macroscopic differential momentum balance for gas flowing in a circular tube can be written as ${ }^{1)}$

$$
\tau_{w} \pi D \cdot d l=-d\left[\frac{\bar{u}^{2}}{\bar{u}}\left(\frac{\pi D^{2}}{4} G\right)+\frac{\pi D^{2}}{4} p\right]
$$

in which $\bar{u}^{2} / \bar{u}$ can be approximated by $\bar{u}$ for turbulent flow while it becomes $(4 / 3) \bar{u}$ for a parabolic velocity distribution. Inserting $\bar{u}=G / \rho_{G}, \quad \tau_{w}=f_{\rho_{G}} \bar{u}^{2} / 2$ and $\rho_{G}=p M / R T$, and integrating under an isothermal condition and accordingly that $f=$ constant, we obtain

$$
2 f G^{2} \frac{l}{D}+\beta \cdot G^{2} \ln \left(\frac{p_{1}}{p_{2}}\right)+\frac{M}{2 R T}\left(p_{2}{ }^{2}-p_{1}^{2}\right)=0
$$

in which $\beta$ is unity and $4 / 3$ respectively for turbulent and laminar flows. This is only a combined equation between those of Dodge (turbulent) ${ }^{2}$ and Schwartzberg et al. (laminar). ${ }^{13)}$

In distillation the vapor flow may undergo nearly an adiabatic process rather than isothermal although mass transfer takes place between the vapor and falling liquid. If Eq. (1) is to be integrated under the adiabatic condition of $p / \rho_{G}{ }^{r}=$ constant, the factor $f$ must be considered a variable and the integrated result becomes considerably complex. Some reasons why the authors adopted the isothermal condition are as follows. (1)

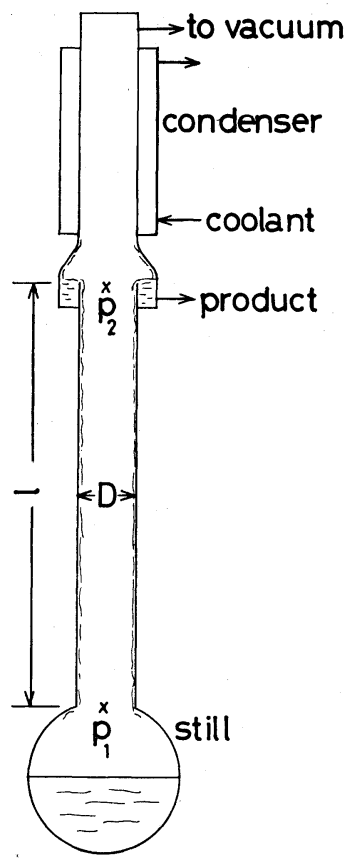

Fig. 1 Illustrative diagram

An equation as simple as possible is desired for the following generalized discussion. (2) The temperature variation in a distillation column is probably not so significant in the absolute temperature scale. (3) Most of the materials treated in vacuum distillation are polyatomic, and then it is expected that $\gamma=c_{p} / c_{v}$ will be nearly unity.

Next in the slip, transition and molecular flow regions, Knudsen's semi-empirical equation may be applied. The original equation ${ }^{9)}$ can be rewritten as

$$
\begin{aligned}
\frac{\pi}{4} D^{2} G & =\left[\frac{\pi}{128} \frac{1}{\mu_{G}} \frac{D^{4}}{l} \rho_{G a v}+\frac{1}{6} \sqrt{2 \pi} \sqrt{\frac{M}{R T}}\right. \\
& \left.\times \frac{D^{3}}{l} \frac{1+\frac{\rho_{G a v}}{\mu_{G}} \sqrt{\frac{R T}{M} D}}{1+1.235 \frac{\rho_{G a v}}{\mu_{G}} \sqrt{\frac{R T}{M}} D}\right]\left(p_{1}-p_{2}\right)
\end{aligned}
$$

in which $\rho_{G a v}$ is to be evaluated at $p_{a v}=\left(p_{1}+p_{2}\right) / 2$. The first term in the right side of Eq.(3) corresponds to the Poiseuille flow rate and when the pressure is reduced it becomes less important compared with the second term which is the molecular flow rate modified empirically.

This equation is correlated to experimental data obtained under conditions of long tube $(l \mid D \gg 1)$ and of somewhat high value in $p_{1} / p_{2}$. Therefore the compressibility effect may be involved in this equation.

\section{Dimensionless Expression of the Pressure Drop Equations}

As is well known the kinetic theory of gases shows 
that the viscosity of a gas is related to the molecular mean speed $\bar{c}(=\sqrt{8 R T / \pi M})$ and the mean free path $\lambda$ as

$$
\mu_{G}=(1 / 2) \rho_{G} \bar{c} \lambda
$$

Using this relation we obtain

$$
\operatorname{Re}_{G}=\frac{D \bar{u} \rho_{G}}{\mu_{G}}=\sqrt{\frac{\pi}{2}} \frac{M a}{K n}
$$

where $M a(=\bar{u} / U)$ is the isothermal Mach number and $K n(=\lambda / D)$ is the Knudsen number which is a measure of the rarefaction. Eqs.(4) and (5) will be used to rearrange the pressure drop equations to dimensionless expressions.

\section{1 Turbulent region}

For simplicity the friction factor $f$ is substituted from the Blasius formula of $0.0791 R e_{G}{ }^{-1 / 4}$, then Eq.(2) becomes

$$
\begin{aligned}
\left(\frac{p_{1}}{p_{2}}\right)^{2}-1= & 0.3164 M a_{2}^{2} \operatorname{Re}_{G}{ }^{-1 / 4} \frac{l}{D} \\
& +2 M a_{2}^{2} \ln \left(\frac{p_{1}}{p_{2}}\right)
\end{aligned}
$$

\section{2 Laminar region}

Substituting $f=16 / R e_{G}$ in Eq.(2) we obtain

$$
\begin{aligned}
\left(\frac{p_{1}}{p_{2}}\right)^{2}-1 & =8 \sqrt{\frac{2}{\pi}} K n_{2} M a_{2} \\
& \times\left[8 \frac{l}{D}+\frac{R e_{G}}{3} \ln \left(\frac{p_{1}}{p_{2}}\right)\right]
\end{aligned}
$$

\section{3 Slip to molecular flow region}

Eq.(3) is rewritten as

$$
\begin{aligned}
& \left(\frac{p_{1}}{p_{2}}\right)^{2}-1=64 \sqrt{\frac{2}{\pi}} K n_{2} M a_{2} \frac{l}{D} / \\
& {\left[1+\frac{256}{3 \pi} \frac{K n_{2}}{\frac{p_{1}}{p_{2}}+1} \frac{K n_{2}+\sqrt{\frac{\pi}{8}\left(\frac{p_{1}}{p_{2}}+1\right)}}{K n_{2}+1.235 \sqrt{\frac{\pi}{8}}\left(\frac{p_{1}}{p_{2}}+1\right)}\right]}
\end{aligned}
$$

In these equations $M a_{2}$ and $K n_{2}$ are to be evaluated at the pressure of $p_{2}$. Eqs. (6) through (8) were calculated and the result is shown in Fig. 2. Ranges assigned to each equation are as follows.

$$
\begin{array}{ll}
\text { Eq.(6): } & R e_{G}>3000 \\
\text { Eq.(7): } & R e_{G}<2000 \text { and } K n_{2}<10^{-2} \\
\text { Eq.(8): } & K n_{2}>10^{-2}
\end{array}
$$

Values calculated from Eqs. (7) and (8) could be connected satisfactorily at the junctions. The quantity $\left[\left(p_{1}-p_{2}\right) / p_{2}\right] /(l / D)$ is determined independently of $l / D$ for low $M a_{2}$ or high $K n_{2}$, but as $M a_{2}$ increases or $K n_{2}$ decreases the curves branch off following various $l / D$ values: Thus to avoid complication only two cases in $l / D$ are shown in Fig. 2. While in this chart the curves drawn with $R e_{G}$ are extended over the whole range of $K n_{2}$. At higher $K n_{2}$, of course, the viscosity in $R e_{G}$ should be defined in the unbounded space and $R e_{G}$ may be considered merely as the parameter $\sqrt{\pi / 2} M a_{2} / K n_{2}$ (see Eq.(5)) or physically as a measure of the reflux flow rate per unit width (see Eq.(13)). It should be noted that the original or physical concept of the Reynolds number is lost in the transition and molecular flow regions.

The sonic velocity limit (SVL) can be considered to be the curve of $M a_{2}=1$. The top pressure limit (TPL) may be illustrated, for example, as follows. When $l / D$ is 100 and the pressure drop limitation is given so that $\Delta p \mid p_{2}<2\left(p_{1} / p_{2}<3\right)$, it becomes clear that $\left(\Delta p / p_{2}\right) /(l / D)$ $<2 \times 10^{-2}$, and therefore we may find that TPL controls when $K n_{2}$ is greater than about $1 \times 10^{-3}$ referring to Fig. 2 (see also Fig. 3).

\section{Flooding Limitation}

A reliable correlation on the flooding in a wetted wall column was given by Kamei et al. ${ }^{7}$, which was reexamined afterwards by Iijima et $a l^{6}{ }^{6)}$ and Fujita ${ }^{4)}$. Although these experiments were performed under conditions of atmospheric pressure and turbulent flow of gases, the correlation may be applicable still at reduced pressures if the gas flow is only continuum and turbulent. By substituting a definition of the reflux ratio, $r=L /(G-L)$, the original flooding equation can be rewritten as

$$
\begin{aligned}
R e_{G} & =198^{0.816}\left(\frac{r+1}{r}\right)^{0.184}\left(\frac{\mu_{L}}{\mu_{G}}\right)^{0.42}\left(\frac{\rho_{G}}{\rho_{L}}\right)^{0.106} \\
& \times\left(\frac{D^{5} \rho_{L}^{3} g^{2}}{\sigma_{L} \mu_{L}^{2}}\right)^{0.188}
\end{aligned}
$$

If one desires to superimpose this $R e_{G}$ upon Fig. 2, the following rearrangement will be available. Considering that the exponent of $\rho_{G}, 0.106$, is small compared with unity, and then putting $\rho_{G}{ }^{0.106} \doteqdot \rho_{G 2}{ }^{0.106}$ $=\left(2 \mu_{G} / c \lambda_{2}\right)^{0.106}$, we can rewrite Eq.(9) as

$$
R e_{G}=Z D^{0.834} \mathrm{Kn}_{\mathrm{2}}^{-0.106}
$$

where

$$
\begin{aligned}
Z= & 198^{0.816} \cdot 2^{0.106}\left(\frac{r+1}{r}\right)^{0.184} \rho_{L}^{0.458} \mu_{L}^{0.044} \\
& \times \sigma_{L}{ }^{-0.188} \bar{c}^{-0.106} \mu_{G^{-0.314}} g^{0.376}
\end{aligned}
$$

Here $Z$ does not depend directly upon the operating pressure, and furthermore its dependence on various materials treated or on variation of the reflux ratio is relatively slight. This range in $Z$ is shown in Table 1 along with roughly assumed ranges of the variables. For example, if we take $Z$ of $3 \times 10^{3}$ and $D$ of $2 \mathrm{~cm}$, a flooding Reynolds number of $5.346 \times 10^{3} \times \mathrm{Kn}_{2}{ }^{-0.106}$ is obtained from Eq.(10) and this is represented in Fig. 3. The asymptote of the flooding line for small $K n_{2}$ can be derived from Eq. (6); the result is

$$
\begin{aligned}
& \left(\frac{p_{1}-p_{2}}{p_{2}} / \frac{l}{D}\right)_{\text {Flooding, Asymptote }} \\
& \quad=8.53 \times 10^{-11} Z^{7 / 4} D^{1.458}\left(10^{5} K n\right)^{1.814}
\end{aligned}
$$

It should be noted that for $Z$ in the range estimated in Table 1 the resulting flooding Reynolds number 


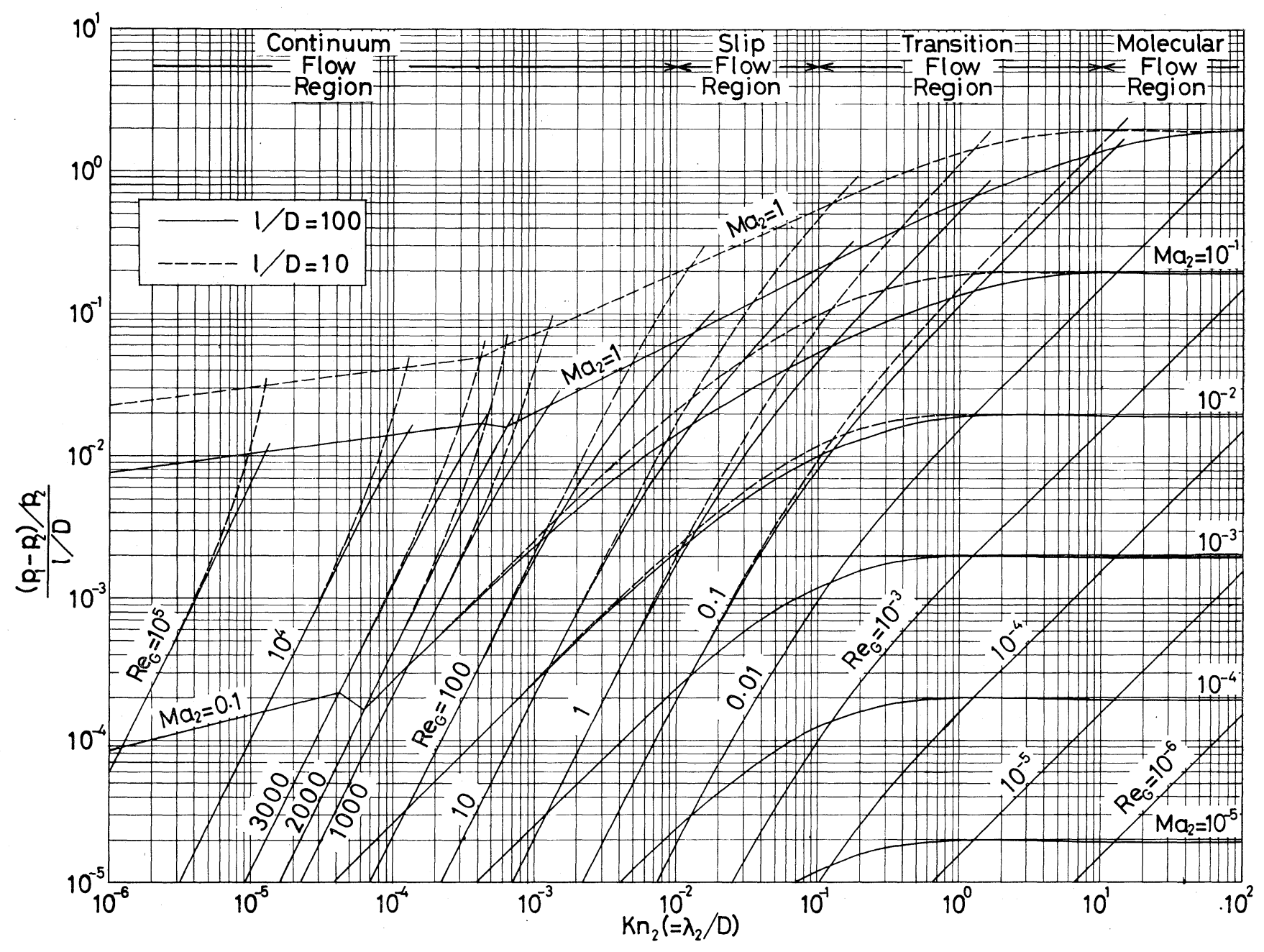

Fig. 2 Results calculated from Eqs. (6) through (8)

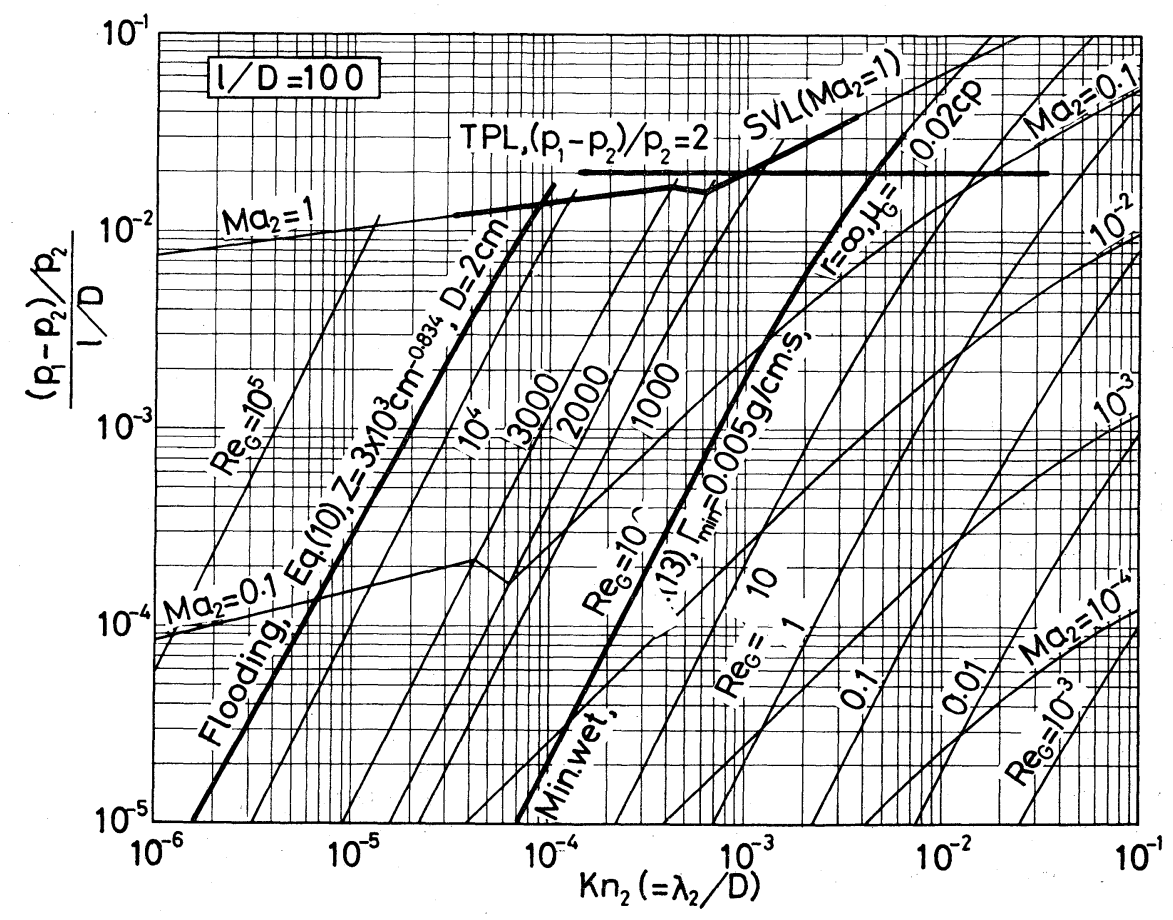

Fig. 3 Four limits based on examples described 


\section{Table 1 Rough estimation of range in $Z$}

\begin{tabular}{llll}
$r$ & $0.5-\infty$ & $\left(\frac{r+1}{r}\right)^{0.184}$ & $1-1.224$ \\
$\rho_{L}$ & $0.7-1.5\left[\mathrm{~g} / \mathrm{cm}^{3}\right]$ & $\rho_{L^{0.458}}$ & $0.894-1.204$ [CGS] \\
$\mu_{L}$ & $0.1-100[\mathrm{cp}]$ & $\mu_{L}^{0.044}$ & $0.738-1$ [CGS] \\
$\sigma_{L}$ & $10-70[\mathrm{dyne} / \mathrm{cm}]$ & $\sigma_{L}{ }^{-0.188}$ & $0.450-0.649[\mathrm{CGS}]$ \\
$T$ & $300-500\left[{ }^{\circ} \mathrm{K}\right]$ & & \\
$M$ & $20-1000[\mathrm{~g} / \mathrm{g}-\mathrm{mole}]$ & & \\
$\bar{c}=\sqrt{\frac{8 R T}{\pi M}}$ & $7.98 \times 10^{3}-7.28 \times 10^{4}[\mathrm{~cm} / \mathrm{sec}]$ & $\bar{c}^{-0.106}$ & $0.306-0.396[\mathrm{CGS}]$ \\
$\mu_{G}$ & $0.01-0.02[\mathrm{cp}]$ & $\mu_{G}{ }^{-0.314}$ & $14.50-18.03[\mathrm{CGS}]$ \\
\hline
\end{tabular}

exceeds 3000 , therefore the flow is turbulent when the tube diameter is greater than $1 \mathrm{~cm}$.

\section{Lower Limit of Vapor Flow Rate Based on the Minimum Wetting Rate of Reflux}

If the flow rate of reflux is insufficient to wet the entire wall surface, operation will become disadvantageous because the dry part does not contribute to mass transfer but increases the pressure drop. If the minimum liquid flow rate, $\Gamma_{\mathrm{min}}$, required to completely wet the wall surface is known, the lower limit of vapor flow rate corresponding to $\Gamma_{\mathrm{min}}$ is written as

$$
\Gamma_{\min }=\frac{1}{\pi D} \frac{\pi D^{2}}{4} G \frac{r}{r+1}=\frac{D}{4} G \frac{r}{r+1}
$$

or

$$
\dot{R} e_{G}=\frac{r+1}{r} \frac{4 \Gamma_{\min }}{\mu_{G}}
$$

Hartley et at. ${ }^{5)}$ and Ponter et al. ${ }^{11)}$ investigated $\Gamma_{\text {min }}$ both theoretically and experimentally, but the results suggest that it is difficult at present to estimate $\Gamma_{\min }$ by a generalized method applicable to various liquids and wall materials.

Furthermore in distillation, the surface tension of the falling liquid varies as it flows down because of both its composition and temperature variations. Also as pointed out by Zuiderweg et al. ${ }^{14)}$, the positive system in which the surface tension increases down the column will result in desirable wetting but the negative system will act in the wrong way. Thus for negative systems it seems that various efforts should be made so that $\Gamma_{\text {min }}$ might be lowered, for example, by the use of surface active agents ${ }^{3)}$ or distributing devices.

While for a positive system there must be a value of $\Gamma_{\text {min }}$ even if the system exhibits good wetting behavior. This value seems to fall out about $0.005 \mathrm{~g} / \mathrm{cm} \cdot \mathrm{sec}$ in preferably wetting systems according to available data $^{10,12)}$ and the authors' experience on vacuum distillation. If this value of $\Gamma_{\min }=0.005 \mathrm{~g} / \mathrm{cm} \cdot \mathrm{sec}$ and the fact that the viscosity of most of gases is in the range of $0.01-0.02 \mathrm{cp}$ are substituted in Eq.(13), the condition of $R e_{G}=100-200$ is required for complete wetting even under total reflux. This criterion is shown also in Fig. 3.

\section{Discussion}

The foregoing criteria for the operating limitations of the falling film column under vacuum distillation are summarized in Fig. 3 based on the examples described individually. The region enclosed by the four limiting lines is considered applicable to operate effectively. In this example, it can be well explained that the upper limit of the vapor throughput is controlled by flooding at higher pressures (at lower Knudsen numbers), and as the pressure is reduced SVL or TPL becomes controlling.

Fig. 3 indicates also that the vapor flow must be laminar at pressures below a value corresponding to the intersection between the lines of $R e_{G}=2000$ and of the upper controlling limit. It has been often stated with only a qualitative explanation that the vapor flow must be laminar at very low pressures ${ }^{8}$. The above observation apparently provides important quantitative support for this statement.

Next the lower limit of the vapor throughput controlled by the minimum wetting rate will be noted. If the corresponding $R e_{G}$ does not reach below 100 as assessed before, $K n_{2}$ will not exceed about $10^{-2}$, and then it will not be necessary to take account of the rarefaction effect on transport phenomena in the vapor phase.

\section{Nomenclature}

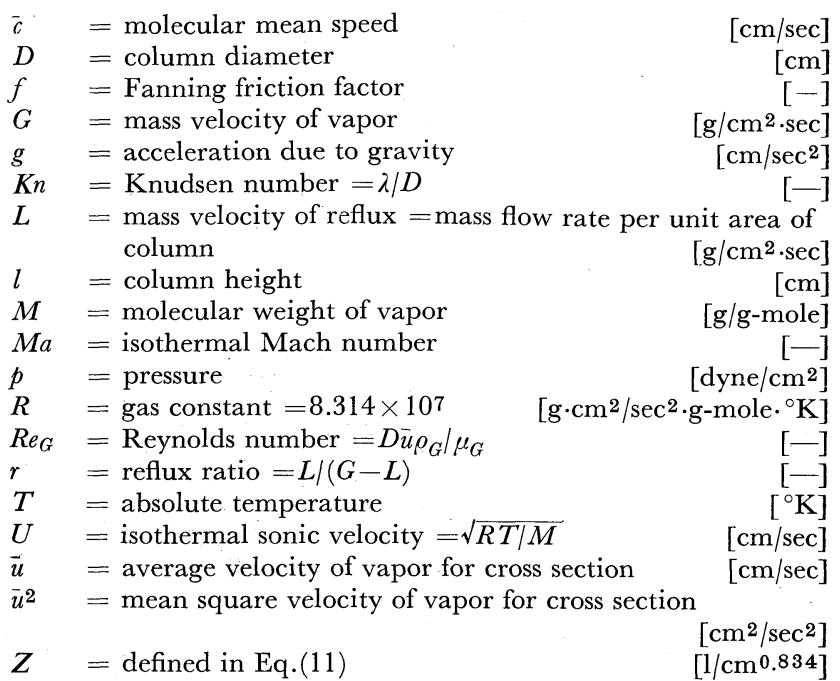


$\beta \quad=$ defined in Eq.(2)

$[-]$

$\Gamma_{\min }=$ minimum wetting rate of falling liquid per unit width of wall

$\lambda=$ molecular mean free path $[\mathrm{g} / \mathrm{cm} \cdot \mathrm{sec}]$

$[\mathrm{cm}]$

$\mu \quad=$ viscosity

$\rho \quad=$ density

$\sigma_{L} \quad=$ surface tension of liquid

$<$ Subscripts $>$

$G \quad=$ for vapor

$=$ for liquid

$=$ at the bottom of column

$=$ at the top of column

$=$ at pressure $p_{a v}\left[=\left(p_{1}+p_{2}\right) / 2\right]$

\section{Literature Gited}

1) Bird, R. B., W. E. Stewart and E. N. Lightfoot: "Transport Phenomena", p. 211, Wiley, New York (1960)

2) Dodge, B. F.: "Chemical Engineering Thermodynamics" p. 350, McGraw-Hill, New York (1944)

3) Francis, R. C. and J. C. Berg: Chem. Eng. Sci., 22, 685 (1967)

4) Fujita, S.: Kagaku Kögaku, 30, 179 (1966)

5) Hartley, D.E. and W. Murgatroyd: Int. J. Heat $\mathcal{E}$ Mass Transfer, 7, 1003 (1964)

6) Iijima, T. and T. Kuzuoka: Kagaku Kōgaku, 31, 1240 (1967)

7) Kamei, S., J. Oishi and T. Okane: Kagaku Kōgaku, 18, 364 (1954)

8) King, R. W.: Brit. Chem. Eng., 12, 722 (1967)

9) Knudsen, M.: Ann. Phys., 28, 75 (1909)

10) Norman, W. S. and D. T. Binns: Trans. Instn. Chem. Engrs., 38, 294 (1960)

11) Ponter, A. B., G. A. Davies, T. K. Ross and P. G. Thornley: Int. J. Heat \& Mass Transfer, 10, 349 (1967)

12) Ponter, A. B., G. A. Davies, W. Beaton and T. K. Ross: Trans. Instn. Chem.Engrs., 45, T345 (1967)

13) Schwartzberg, H. G. and M. Gurevich: A. I. Ch. E. Journal, 16, $762(1970)$

14) Zuiderweg, F.J. and A. Harmens: Chem. Eng. Sci., 9, 89 (1958)

\title{
DISTILLATION CALCULATION BY THE MODIFIED PERTURBATION METHOD*
}

\author{
Yasuo HIROSE, Hitoshi HIRAIWA AND \\ JiRo SUDOH \\ Department of Chemical Engineering, Tokyo Metropolitan \\ University, Tokyo, Japan
}

\begin{abstract}
An unique perturbation method for the distillation calculation of a non-ideal system was proposed by Acrivos and Amundson. This method, however, is limited only to the first-order perturbation and gives an approximate solution, for the equation with the second and further perturbed terms is too complicated to use for practical purposes. To improve this point the Double Perturbation Method is presented here. Although the equations used in the presented method are obtained by the same reasoning as the above authors, this method, whose tiresome procedures can be overcome by computer, gives the strict solution.
\end{abstract}

\section{Introduction}

An interesting perturbation method for the distillation calculation was proposed by Acrivos and Amundson ${ }^{1,2)}$. In their procedure, at first a nonperturbed distribution profile is obtained and next the first-order perturbed terms are calculated. The nonperturbed distribution profile is corrected by these first-order perturbed terms. From a mathematical viewpoint, this method is very interesting and moreover implies further application. The solution, however, sometimes remains an approximate one, for the secondorder term and further terms are truncated. If these

\footnotetext{
* Received on April 19, 1972

Presented at the 37th Annual Meeting of the Soc. of Chem. Engrs., Japan, on the 5th of April, 1972
}

terms were considered, from the practical computational stand-point, the procedures would be too complicated to carry out the calculation as Acrivos and Amundson described it in their paper. The equations used in the following method are derived by the same reasoning as the above authors.

The equations concerning an enriching section of a column have been presented by them. But for a stripping section only the final equations of the nonperturbed case are presented in their work.

The strict solution can be obtained by repeated application of a multi-usage of the first-order perturbation, the detailed explanation of which will be described in a later section.

\section{The Basic Equations for the Stripping Section of a Rectification Column}

